\title{
Identificación y Ajuste Paramétrico de una Máquina Trifásica de Inducción Magnética usando Algoritmos Genéticos
}

\author{
Leopoldo Simón y José M. Monzón \\ Departamento de Ingeniería Eléctrica, Universidad de Las Palmas de Gran Canaria, Islas Canarias- \\ España (e-mail: Isimon@pas.ulpgc.es)
}

Recibido Oct. 29, 2009; Aceptado Ene. 18, 2010; Versión Final recibida Feb. 12, 2010

\begin{abstract}
Resumen
En este artículo se analiza mediante el método de los elementos finitos un motor trifásico de inducción magnética, al cual se le han aplicado diferentes geometrías de barras en el rotor. Estas variaciones geométricas del rotor crean un perfil electromagnético diferente para cada tipo de geometría. Para estudiar el rotor en movimiento, se procede con un análisis en frecuencia del modelo que permitirá simular la evolución de la máquina eléctrica, tanto del modelo circuital como del modelo continuo por elementos finitos. Los parámetros identificativos deducidos del método de los elementos finitos, se ajustaron mediante algoritmos genéticos evolutivos. De esta forma, los parámetros en el modelo circuital quedan identificados para cada geometría objeto de estudio. Se hace también una comprobación del par como validación del modelo, comprobando y verificando la exactitud del método.
\end{abstract}

Palabras clave: elementos finitos, análisis en frecuencia, parámetros identificativos, algoritmos genéticos

\section{Identification and Parametric Adjustment of a Three- Phase Induction Machine with Genetic Algorithms}

\begin{abstract}
In this paper the finite element method is applied to the analysis of a three-phase magnetic induction motor squirrel-cage. Different geometries have been applied in the design of the rotor. These geometric variations of the rotor create an electromagnetic profile for each type of geometry. To study the rotor motion, a frequency analysis of the model has been done. The identifying parameters deduced from the finite element method, are adjusted by evolutionary genetic algorithms. Thus, the parameters in the circuit model were identified for each geometry under consideration. A check of the torque was done to validate the model, verifying the accuracy of the method.
\end{abstract}

Keywords: finite element, frequency analysis, identifying parameters, genetic algorithms 


\section{INTRODUCCIÓN}

Las herramientas informáticas han permitido llevar a cabo técnicas numéricas que antaño eran irrealizables, como son la simulación de modelos circuitales equivalentes basados en sus ecuaciones de equilibrio (Fig. 3), y métodos de modelado discreto como el Método de los Elementos Finitos (MEF), conocidos desde hace bastante tiempo, pero sólo aplicables a partir de la aparición de estas poderosas herramientas de cómputo (Meeker, 2002; Mirafzal et al., 2009; Nerg et al., 2004; Yamazaki, 2002).

No solo es interesante la identificación y el control en las máquinas de inducción, ya sea para auditorías o modelado constructivo previo, sino que también es necesaria su optimización ante la variedad de procesos industriales existentes, que particularizan distintos diseños de las mismas. La aportación de este artículo está en el ajuste por algoritmos genéticos (AG) de los parámetros del modelo circuital (Meeker, 2002; Mirafzal et al., 2009) de un motor trifásico de inducción magnética, con diferentes geometrías en el diseño del rotor (Fig. 1), minimizando el error cuadrático de los resultados obtenidos con el modelo continuo MEF, para las distintas geometrías.

El MEF, es un método de aproximación de problemas continuos (Belmans et al., 1990; Nerg et al., 2004; Salon, 1995). Su planteamiento se basa en la transformación de un cuerpo de naturaleza continua en un modelo discreto aproximado. Esta transformación es denominada como discretización del modelo. El conocimiento de lo que sucede en el interior de este modelo del cuerpo aproximado, se obtiene mediante la interpolación de los valores conocidos en los nodos. Es por tanto una aproximación de los valores de una función a partir del conocimiento de un número determinado y finito de puntos.

Paralelamente a la evolución tecnológica que ha permitido el diseño de potentes herramientas electrónicas de cálculo, han evolucionado en complejidad y abstracción los algoritmos para resolución de multitud de problemas científicos. Los algoritmos genéticos son una prueba de ello (Çunkas y Akkaya, 2006; Rasmus, 2003; Von Lücken Martínez, 2003). Una vez más, un proceso natural como la selección y la evolución de los individuos en una población, ha sido aplicado al campo científico para hacer evolucionar sistemas y observar su tendencia, a partir de ciertas condiciones iniciales. Los individuos en las poblaciones intercambian información por medio de operadores evolutivos.

Existen tres operadores genéticos principales: la selección, el cruzamiento y la mutación (Çunkas y Akkaya, 2006). Mediante la selección, los individuos son escogidos de la población de acuerdo al valor de su función de adaptación, para someterse a la acción futura de otros operadores. El operador de cruzamiento se encargará de intercambiar los componentes de los individuos seleccionados para producir nuevas soluciones, encargándose de la transferencia por herencia de las características de los mejores individuos de una generación a la siguiente. La mutación es utilizada como un operador cuyo propósito es la exploración aleatoria de nuevas porciones del espacio de búsqueda, introduciendo nuevo material genético en la búsqueda de soluciones. La ventaja principal de estos algoritmos sobre otros métodos de optimización, es que son capaces de encontrar un extremo de la función global o absoluto, en lugar de uno local, y que la estimación inicial no tiene que estar cerca de los valores reales. Más aún, estos trabajan directamente sobre la función y no requieren el uso de su derivada ni de ninguna otra función auxiliar.

Toda esta metodología aplicada al estudio de máquinas de inducción, permite un nuevo punto de vista en el conocimiento profundo de las mismas. El programa utilizado como aplicación del método por elementos finitos es el Finite Element Method Magnetics (FEMM), que es una herramienta de software libre muy versátil (Meeker, 2002). Para el análisis por algoritmos genéticos se han utilizado las librerías de código abierto GAlib, escritas por Matthew Wall en el Massachusetts Institute of Technology (MIT), que contiene un conjunto de algoritmos genéticos objeto en C++ (Wall, 2000).

El artículo en su conjunto ha sido elaborado con software libre y/o de código abierto, en el procesador de texto para documentos científicos Latex, y bajo el sistema operativo openSUSE-Linux. 


\section{MODELO CONTINUO FEM}

En este apartado se hace un análisis de varias geometrías de barras en el rotor (Fig. 1), mediante el programa FEMM que ensambla todas las ecuaciones de campo para el caso magnético, en el estudio de máquinas eléctricas.
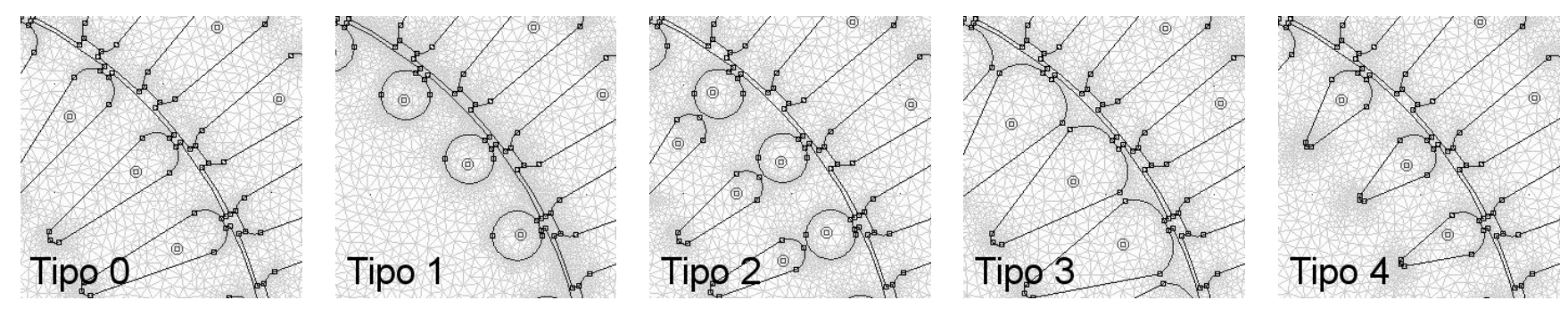

Fig. 1: Sección de los cinco tipos de geometrías de barras de rotor analizadas.

La densidad de corriente $\vec{J}$, está definida de tal forma que su orientación es perpendicular al plano, con lo cual, el campo vectorial $\vec{A}$, que es un vector de tres componentes y que tiene su misma orientación, queda definido como una función de $A_{z}(x, y)$. Con lo cual, un problema que en principio tenía un planteamiento tridimensional, al tener simetría plana se convierte en un problema bidimensional (Molinar et al., 1996).

Para la derivación de las ecuaciones de elementos finitos en su aplicación para las máquinas eléctricas en dos dimensiones, entre los métodos existentes es bastante utilizado el método de Galerkin, que es un caso especial del método de los residuos ponderados, donde la función de ponderación tiene la misma forma que la función de forma del elemento finito (Salon, 1995).

En (1) se muestra la forma armónica de la ecuación de difusión del vector potencial magnético, para un problema en dos dimensiones, donde su componente $z$ es desconocida.

$(\nabla \times \vec{H})=\nabla \times\left[\frac{1}{\mu}\left(\nabla \times \vec{A}_{z}\right)\right]=\vec{J}_{z}$

En dicha ecuación $\nabla \times$ es el operador diferencial rotacional, y se define $\vec{A}_{z}(\mathrm{~Wb} / \mathrm{m})$, como el vector potencial magnético, $\vec{J}_{z}\left(\mathrm{~A} / \mathrm{m}^{2}\right)$ como la densidad de corriente, $\vec{H}(\mathrm{~A} / \mathrm{m})$ la intensidad del campo magnético y $\mu(\mathrm{H} / \mathrm{m})$ la permeabilidad magnética del medio.

Si la excitación es en el dominio de la frecuencia y los materiales son lineales, con una simetría plana esta última expresión se simplifica quedando como se muestra en (2)

$\nabla \cdot v \nabla A_{z}-\sigma j \omega A_{z}=-J_{z}$

donde el operador nabla $\nabla \cdot$, representa el operador divergencia y el operador $\nabla$ el gradiente, y donde $v$ es la reluctividad del medio que es la inversa de la permeabilidad $\mu$, la $\omega(1 / \mathrm{s})$ es la frecuencia angular, $\sigma$ (siemens $/ \mathrm{m}$ ) es la conductividad eléctrica, y $A_{z}, J_{z}$ son las componentes normales del vector potencial magnético y la densidad de corriente respectivamente. El operador nabla $\nabla \cdot$, representa el operador divergencia y el operador $\nabla$ el gradiente (Çunkas et al., 2006). 


\section{CONDICIONES DE FRONTERA DEL MODELO CONTINUO}

La aplicación del MEF, implica por naturaleza un dominio finito. Los problemas sin frontera o de frontera abierta, se enfocan mediante las transformaciones de Kelvin (Molinar et al., 1996; Salon, 1995). Para ello, la región sin frontera se mapea dentro de una región limitada circular con frontera, donde los problemas sean más fácilmente resueltos.

En el caso de dos dimensiones, el exterior, puede ser modelado por otra región también circular, que representa el campo lejano, donde en su centro, el valor del potencial $A=0$. A los bordes de los círculos que limitan ambas regiones se les imponen condiciones de frontera periódicas. Con ello se consigue forzar la continuidad de $A$ en los bordes de ambas regiones. Por otra parte, con la finalidad de hacer más eficiente el estudio, se ha reducido los análisis al primer cuadrante de la sección de la máquina. Los valores de distribución en el primer cuadrante, se ha comprobado que equivalen a la cuarta parte de los valores totales que se determinan en la sección completa (Fig. 2).
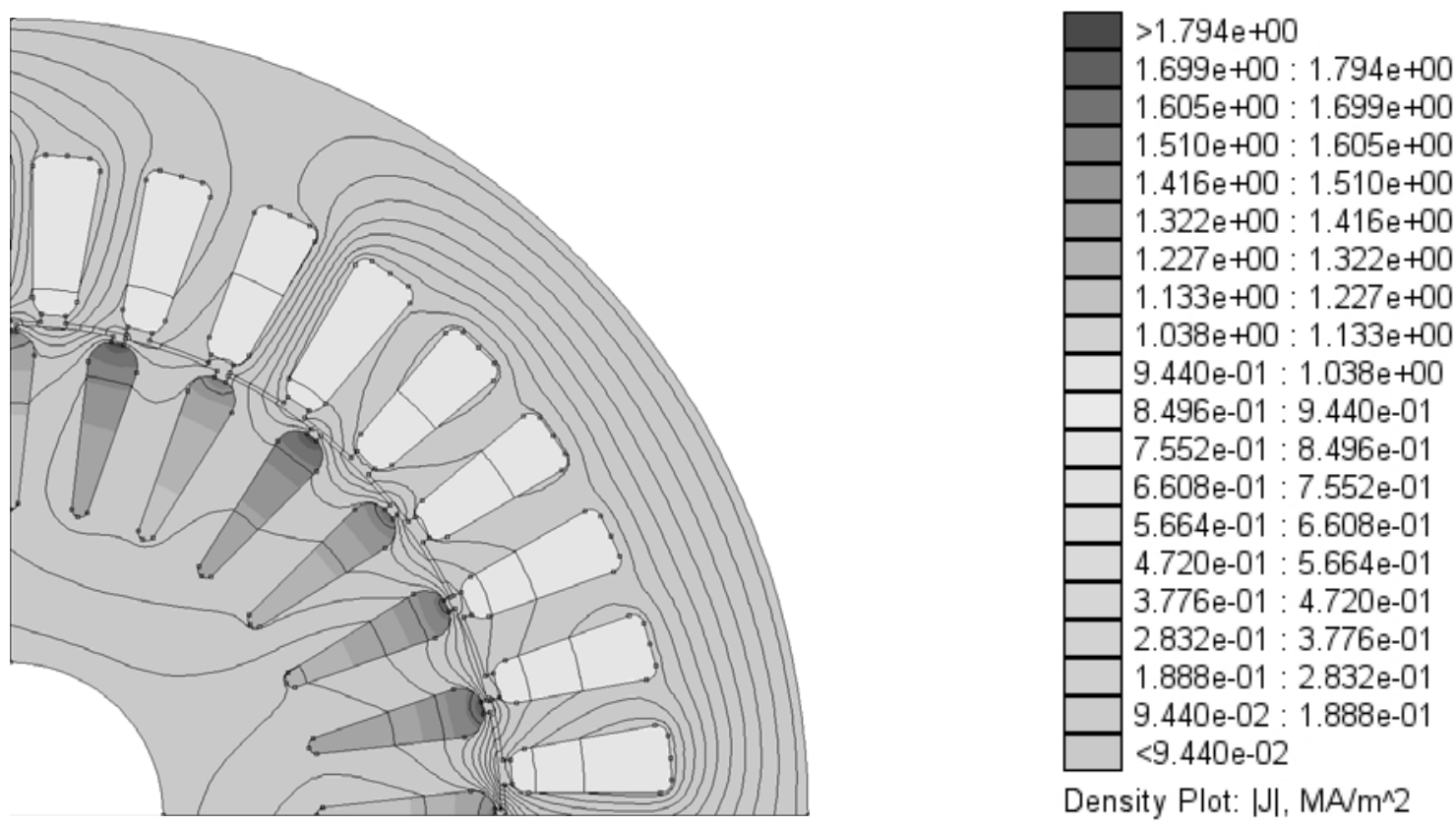

Fig. 2: Módulo de la densidad de corriente en el modelo continuo por elementos finitos.

Para ello ha de tenerse en cuenta que las condiciones de contorno en ambos lados adyacentes a los otros cuadrantes, deberán ser antiperiódicas. Ello reducirá enormemente los tiempos y los costes computacionales. Por tanto, todos los análisis están reducidos al primer cuadrante (Fig. 2).

\section{IDENTIFICACIÓN DEL MODELO PARAMÉTRICO}

Una máquina de inducción con un rotor en movimiento puede ser modelada usando un modelo circuital relativamente simple (Belmans et al., 1990; Çunkas et al., 2006; Meeker, 2002; Mirafzal et al., 2009). Aunque los parámetros circuitales pueden ser a menudo aproximados por expresiones de forma en términos explícitos en cuanto a la geometría del motor, la identificación de estos parámetros mediante el análisis por el MEF es validar las aproximaciones y simplificaciones que inevitablemente tienen que ser hechas en la derivación de las fórmulas de diseño analíticas.

Para identificar parámetros en un modelo de motor de inducción, se necesita un modelo razonablemente sencillo que podría ser el mostrado en la Fig. 3.

En este modelo, el flujo de dispersión está agrupado en el lado del estátor, en la impedancia $L_{l}$ (Boglietti et al., 2007). La inductancia $M$ es la inductancia mutua entre el rotor y el estátor. 
Representado por $R_{r}\left(\omega / \omega_{s}\right)$ se tiene el trabajo disipado en forma de calor en el rotor y en la carga como potencia mecánica.

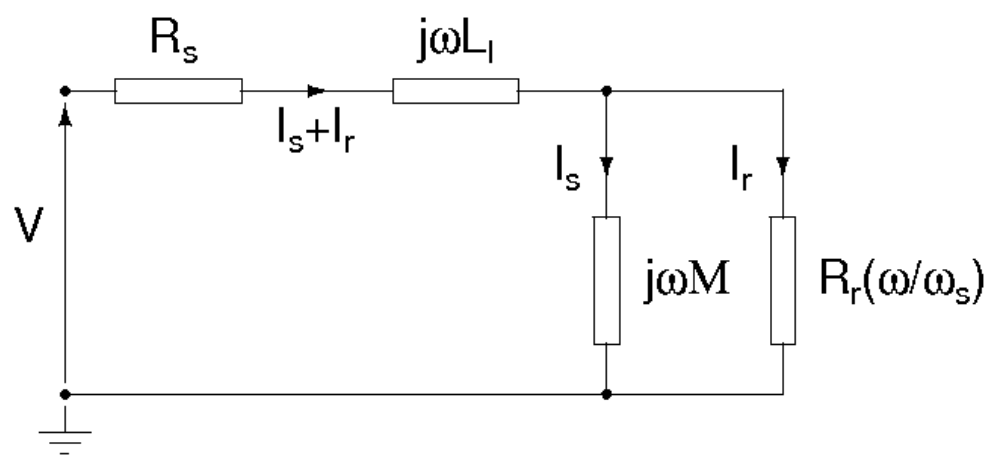

Fig. 3: Modelo simple por fase del motor de inducción en régimen estacionario.

Si $\omega$ es la frecuencia angular en radianes/segundo y $p$ es el número de pares de polos, la frecuencia de deslizamiento $\omega_{s}$ en términos de frecuencia eléctrica y velocidad mecánica de rotor $\omega_{r}$, queda definida en (3) de la siguiente forma

$\omega_{s}=\omega-p \omega_{r}$

o sea, como la diferencia entre la frecuencia eléctrica y la frecuencia mecánica del rotor.

Como relaciones útiles se obtienen la inductancia dependiente de la frecuencia de deslizamiento (4), mostrándose el resultado en sus componentes real y compleja

$L\left(\omega_{s}\right)=\left(L_{l}+\frac{M}{1+\left(\tau \omega_{s}\right)^{2}}\right)-j\left(\frac{\tau \omega_{s} M}{1+\left(\tau \omega_{s}\right)^{2}}\right)$

La dependencia de $L\left(\omega_{s}\right)$ sobre la frecuencia de deslizamiento permitirá identificar algunos parámetros del motor como la inductancia mutua $M$, la inductancia de dispersión $L_{l}$ y la resistencia del rotor $R_{r}$ (Mirafzal et al., 2009), siendo $\tau$ la constante de tiempo del rotor definida como $M / R_{r}$.

Como validación del modelo se obtiene la expresión (5), perfectamente válida para el par electromagnético $T$.

$T=3 p M i^{2}\left(\frac{\tau \omega_{s}}{1+\left(\tau \omega_{s}\right)^{2}}\right)$

Si la corriente se toma como constante y la frecuencia de deslizamiento se varía, se obtiene una curva con un par máximo en $\tau \omega_{s}=1$.

\section{ANÁLISIS MEDIANTE EL FEMM}

Al usar el FEMM, se analiza el rotor de forma estática. En el caso de velocidad cero, el deslizamiento de frecuencia simplemente degenera a $\omega_{s}=\omega$.

Lo lógico sería hacer la identificación de los parámetros en base a los resultados de par $T$ analizados, usando una corriente de estátor constante sobre un rango de frecuencias, simulando así el movimiento del rotor. Pero entonces el cálculo del par debe hacerse mediante el tensor de Maxwell (6), lo que es menos preciso en los desarrollos integrales que incorpora el programa FEMM. 
La densidad de fuerza del tensor de Maxwell en $\mathrm{N} / \mathrm{m}^{2}$ para un motor circular queda definida por las componentes normal y tangencial de la densidad de flujo (6).

$$
\begin{aligned}
& p_{t}=\frac{B_{n} B_{t}}{\mu_{0}} \\
& p_{n}=\frac{B_{n}^{2}-B_{t}^{2}}{2 \mu_{0}}
\end{aligned}
$$

El modulo de la densidad de flujo es $|B|=\sqrt{B_{n}^{2}+B_{t}^{2}}$, como se encuentra representado en la Fig. 8. Entonces, el par electromagnético en $\mathrm{N}^{*} \mathrm{~m}$, a lo largo del entrehierro queda definido como $T=\oint r\left(\frac{B_{n} B_{t}}{\mu_{0}}\right) d s$.

Sin embargo, otra opción sería mediante el ajuste de los resultados de inductancia que se obtienen por el análisis del bloque integral $\bar{A} \cdot \bar{J}$, formado por el potencial vector magnético y la densidad de corriente sobre el volumen del bobinado de interés, en el rango de frecuencias analizado (Nerg et al., 2004; Phumiphak y Chat-Uthai, 2002). De esta forma se obtiene una alta precisión, ya que la integral de volumen (7) está estrechamente relacionada con la energía almacenada.

$$
L=\int \frac{\bar{A} \cdot \bar{J}}{|i|^{2}} d v
$$

\section{AJUSTE DE LOS PARÁMETROS POR ALGORITMOS GENÉTICOS}

Se han obtenido en primer lugar, los resultados analíticos del modelo para los cinco tipos de geometrías del rotor propuestas (Fig. 1), y en segundo lugar, los resultados de las simulaciones hechas mediante el análisis por elementos finitos mediante el FEMM. Los resultados de ambos análisis han dado un perfil paramétrico de la máquina de inducción para cada geometría objeto de estudio.

En el análisis en frecuencia realizado para simular el movimiento del rotor, se ha obtenido la evolución de algunos parámetros significativos por fase como son el flujo y el par, desde el tensor de Maxwell (6) calculado en el FEMM para diferentes frecuencias de deslizamiento, con lo que se podrá identificar los parámetros $M, L_{l}$, y $\tau$ del motor de inducción.

Estos parámetros se han ajustado con AG, tomando como función objetivo (8) la parte real e imaginaria de la inductancia como función dependiente de la frecuencia de deslizamiento, estrechamente ligada con estos parámetros de ajuste. El procedimiento del AG consiste en hacer mínimo el error cuadrático medio entre los valores obtenidos en las simulaciones en frecuencia mediante el FEMM (7), y los valores obtenidos del circuito equivalente (4) para cada geometría.

$$
E_{\text {objectivo }}=\sum_{k=0}^{10}\left(\operatorname{Re}\left[\int \frac{\overline{A^{k} \cdot \overline{J^{k}}}}{|i|^{2}} d v\right]-\left[L_{l}+\frac{M}{1+\left(\tau \omega_{s}^{k}\right)^{2}}\right]\right)^{2}
$$

Para la parte imaginaria de la inducción, se utiliza la misma expresión anterior (8). En el AG ha sido utilizado un genoma binario a decimal con 16 bits de resolución (Çunkas y Akkaya, 2006; Von Lücken Martínez, 2003). El algoritmo obtiene un valor óptimo para los parámetros $M, L_{l}$, y $\tau$. Para el ajuste de los tipos de geometría estudiados, los valores de los operadores evolutivos de selección, cruzamiento y mutación, que permitieron un ajuste óptimo, se indican en la Tabla 1. 


\section{RESULTADOS Y DISCUSIÓN}

Los valores generales aplicados en el algoritmo genético son los mismos, y se basan en un tamaño de población $=50$, un número de generaciones $=200$, una probabilidad de mutación de 0,01 y una probabilidad de cruzamiento $=0,6$.

Tabla 1: Valores óptimos resultantes de la solución por algoritmos genéticos de la constante de tiempo del rotor $\tau(\mathrm{s})$, la inductancia mutua $M(\mathrm{H})$ y la inductancia de dispersión $L_{l}(\mathrm{H})$, para los cinco tipos de geometría.

\begin{tabular}{|c|c|c|c|c|c|}
\hline & Tipo 0 & Tipo 1 & Tipo 2 & Tipo 3 & Tipo 4 \\
\hline$\tau(s)$ & 0,1635770 & 0,0703136 & 0,0619364 & 0,238499 & 0,0944839 \\
\hline$M(H)$ & 0,3131150 & 0,1646140 & 0,1402150 & 0,139849 & 0,1640650 \\
\hline$L_{I}(H)$ & 0,0155642 & 0,0068207 & 0,0093843 & 0,006775 & 0,0088807 \\
\hline
\end{tabular}

Como ejemplo, se representan en la Fig.4 y en la Fig. 5 los ajustes obtenidos para la geometría tipo 0 , donde los valores resultantes del ajuste por AG han proporcionado unos valores para $\tau=0,163577$ (s), $M=0,313115(\mathrm{H}), L_{1}=0,015564(\mathrm{H})$.

La representación muestra la parte real (Fig. 4) y la parte imaginaria (Fig. 5) de la inductancia $L(H)$ del modelo circuital ajustada con el modelo continuo simulado por el MEF. Para ello se ha hecho un análisis en frecuencia, variando la frecuencia de deslizamiento desde 0 hasta $10 \mathrm{~Hz}$.

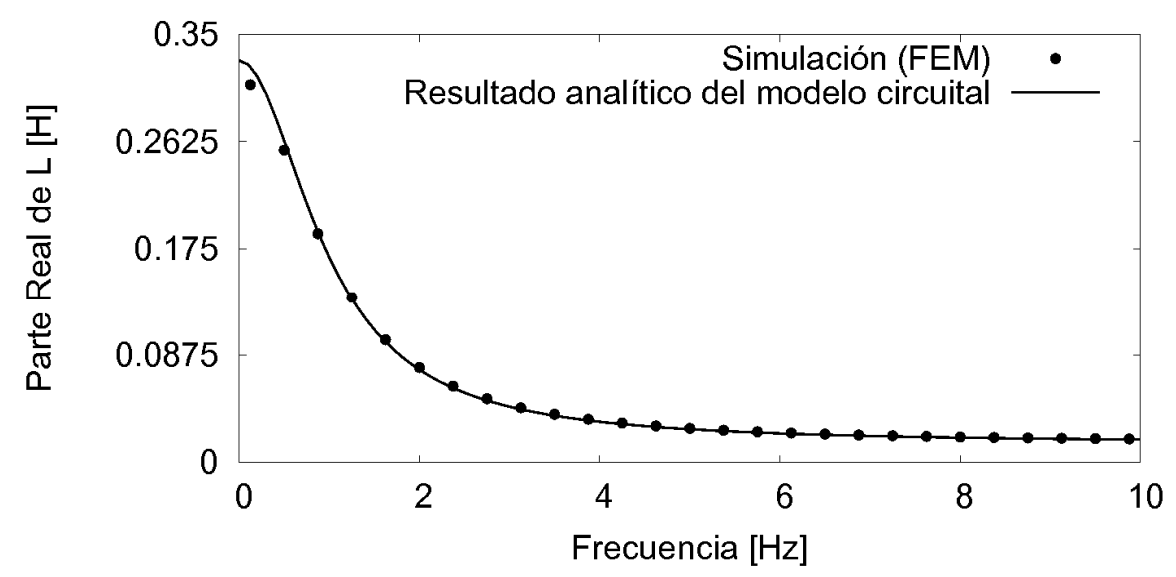

Fig. 4: Ajuste de la parte real de la inducción mediante AG para la geometría de rotor tipo 0.

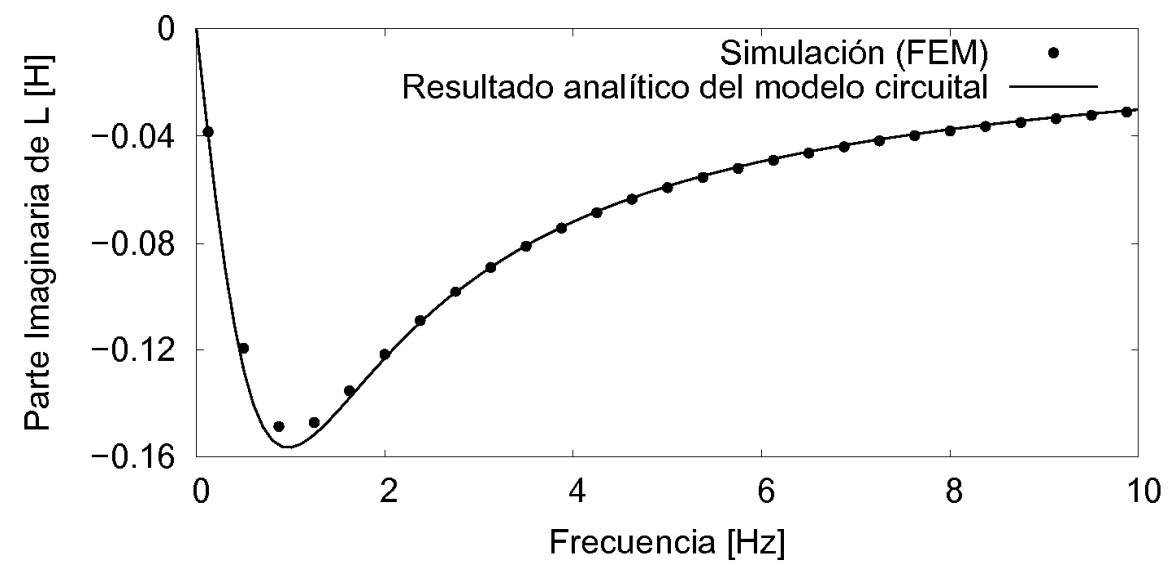

Fig. 5: Ajuste de la parte imaginaria de la inducción mediante AG para la geometría de rotor tipo 0. 
Los valores que permitieron este ajuste están recogidos en la Tabla 1, junto con los valores que ajustaron las demás geometrías. Estos valores determinan por su relación, el flujo concatenado con cualquier fase y el par como una función de corriente de fase.

Como validación del procedimiento, se ha representado (Fig. 6) el par electromagnético entre el modelo circuital paramétrico y el tensor de Maxwell del MEF para la geometría tipo 0 , donde el ajuste obtenido manifiesta la fiabilidad del método. Las simulaciones con el MEF dan el par electromagnético utilizando el tensor magnético de Maxwell, y este es validado con el par electromagnético del modelo paramétrico (5).

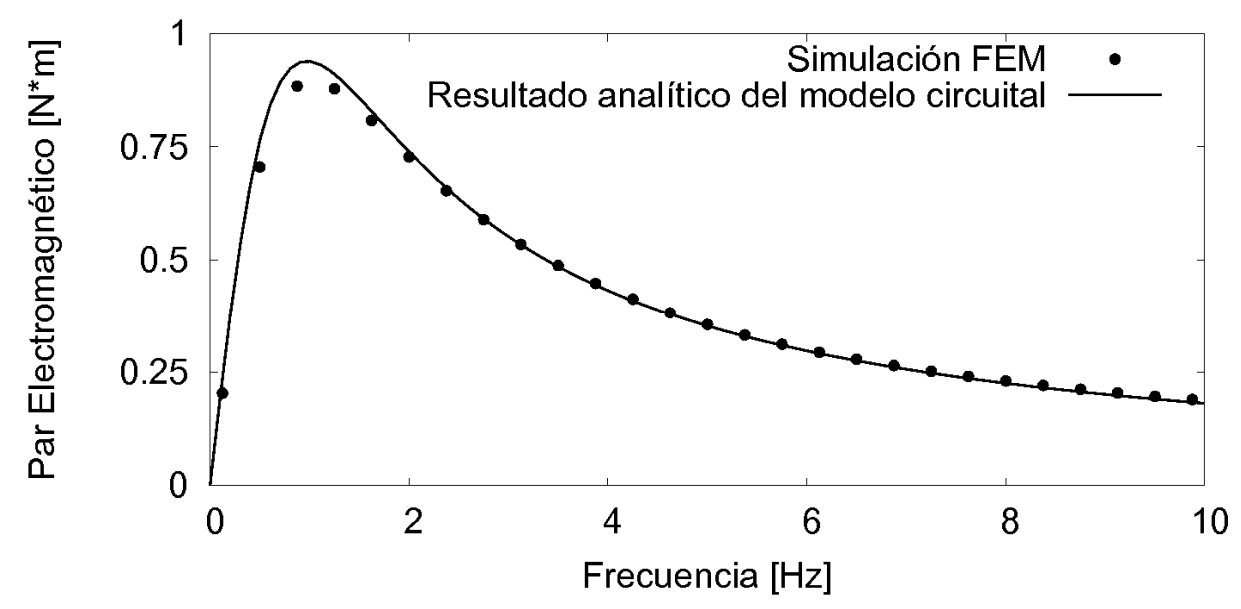

Fig. 6: Validación del par electromagnético para la geometría de rotor tipo 0.

La Fig. 7 muestra el módulo del vector potencial magnético, junto a sus partes real e imaginaria. La representación se muestra a lo largo del entrehierro en el primer cuadrante. La longitud del entrehierro es de $65 \mathrm{~mm}$ en un cuadrante.

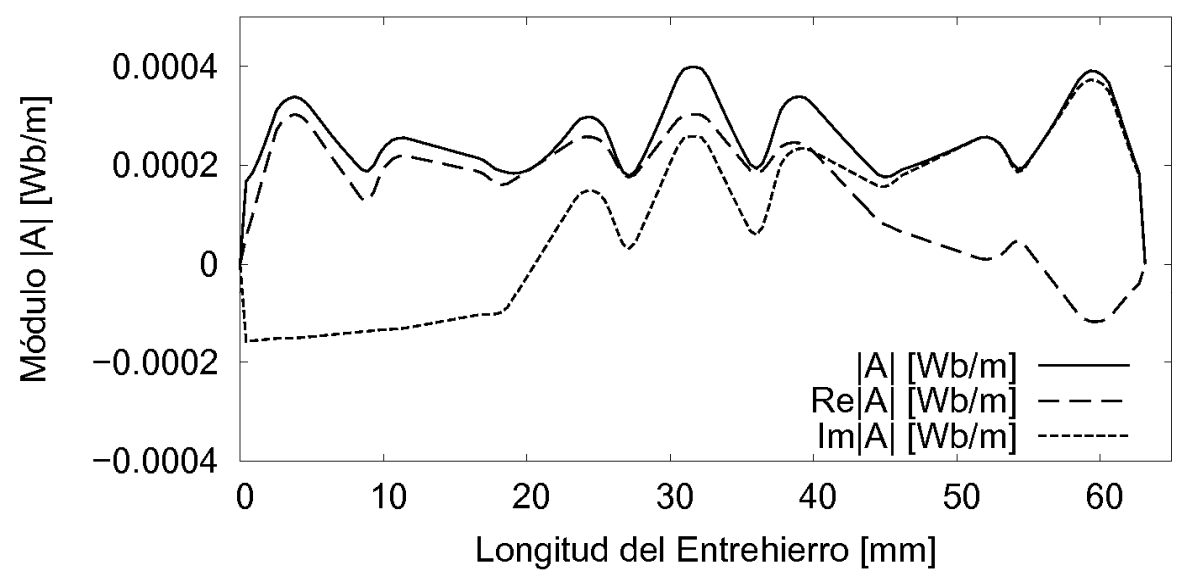

Fig. 7: Perfil del módulo del vector potencial magnético y sus componentes complejas.

La Fig. 8 muestra el perfil del módulo de la inducción magnética a lo largo del entrehierro en el primer cuadrante. Estos valores son necesarios para obtener el par electromagnético desde el tensor de Maxwell. La relación entre la Fig. 7 y la Fig. 8 viene dada por $\bar{B}=\nabla \times \bar{A}$.

Como parte de los resultados, se muestra una representación en la Fig. 9 de las curvas características de par-velocidad de todas las geometrías aplicadas a las barras del rotor que se han estudiado. 


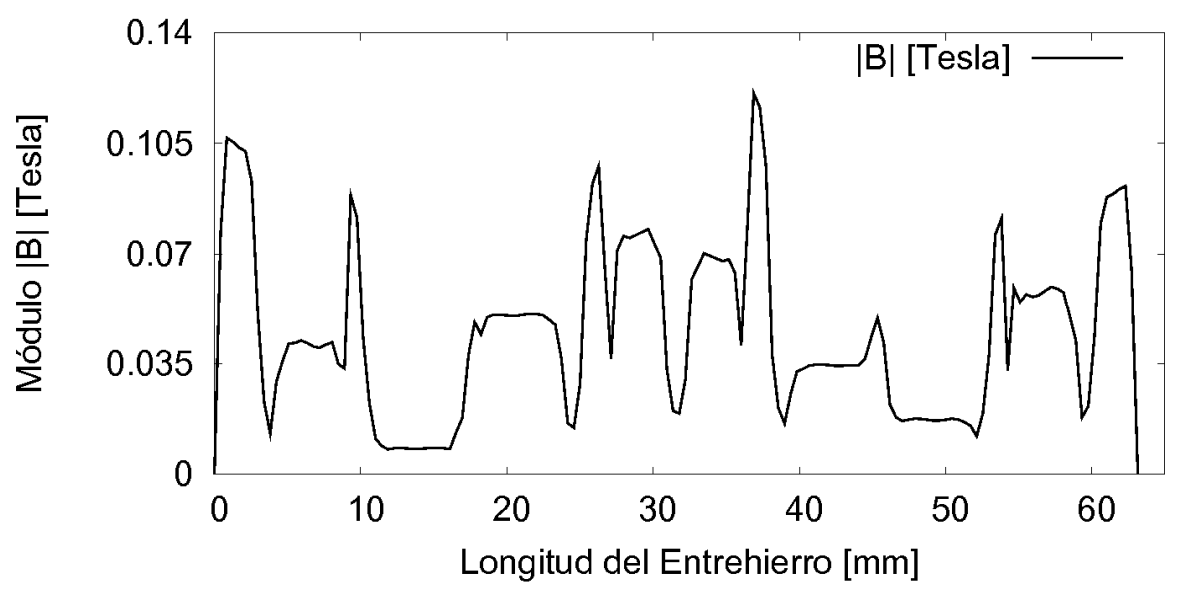

Fig. 8: Módulo de la inducción magnética.

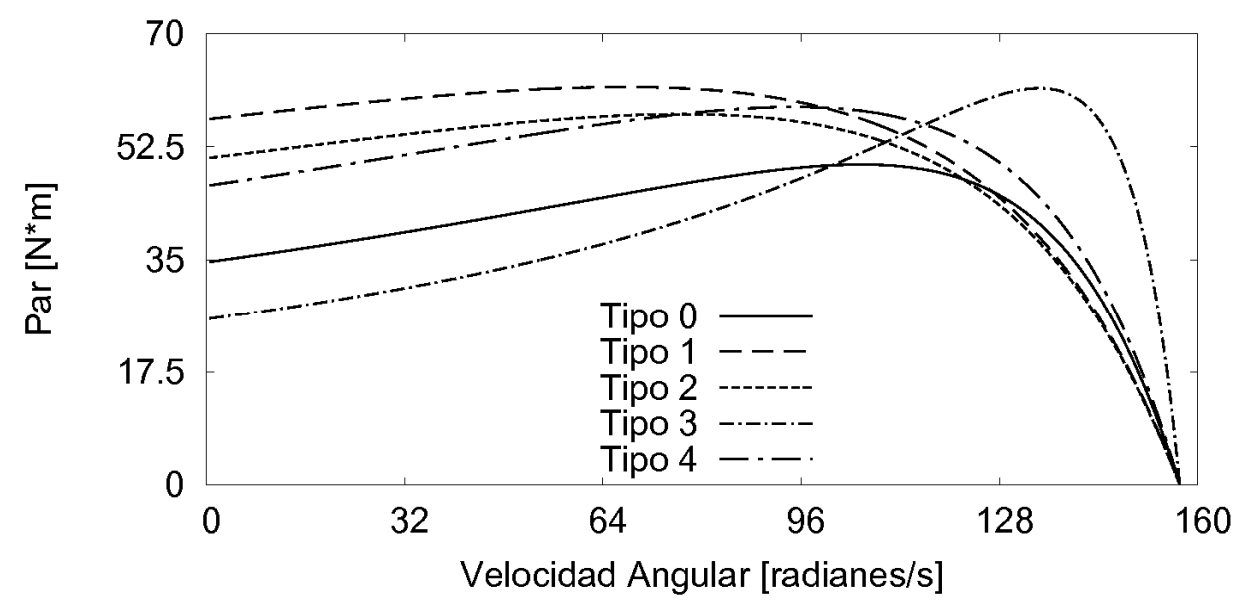

Fig. 9: Representación de las curvas par-velocidad para todos los tipos de geometría.

Así mismo, en la Fig. 10 se observan los diferentes valores de la intensidad en una de las fases para todas las geometrías estudiadas.

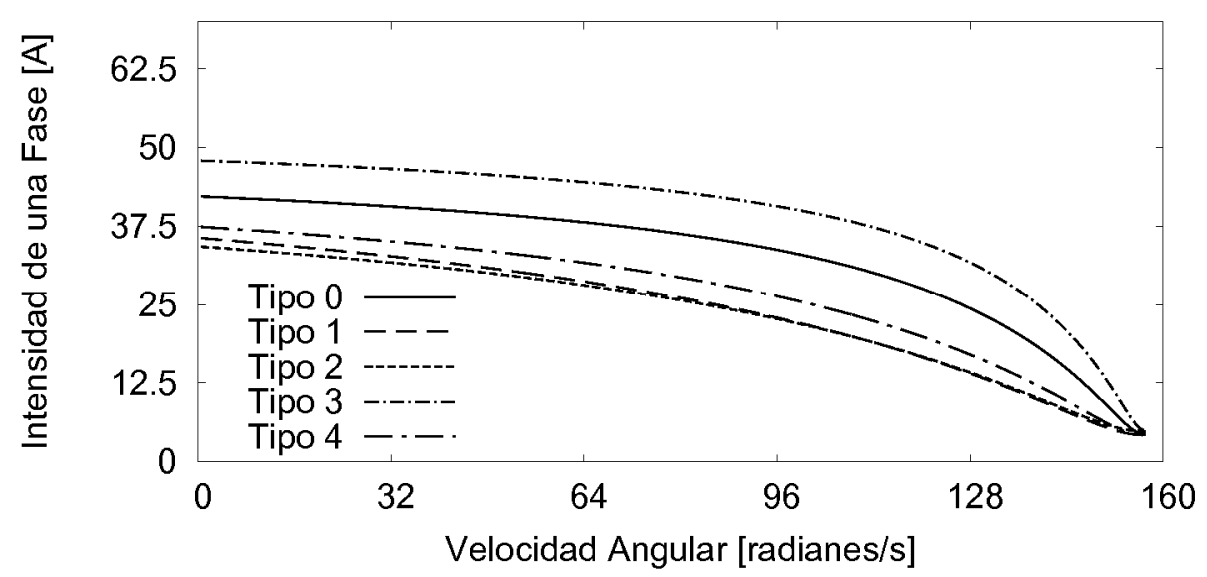

Fig. 10: Representación de la intensidad en una de las fases, para todas las geometrías. 
Estas curvas se han obtenido mediante un programa realizado en Scilab, donde se han incluido todos los valores de ajustes por AG mostrados en la Tabla 1.

\section{CONCLUSIONES}

Se ha conseguido el ajuste por AG de los parámetros del modelo circuital de un motor trifásico de inducción magnética con el modelo continuo MEF, para las geometrías propuestas. Para ello, se ha creado un circuito equivalente de una fase de la máquina, operando en régimen permanente y en estado de equilibrio. Se han identificado los parámetros en base a los resultados de la inductancia, por su precisión. El método utilizado para la identificación, es un análisis en frecuencia del modelo por elementos finitos y del modelo circuital. Se ha utilizado como método de optimización los algoritmos genéticos evolutivos, por su robustez y precisión. Mediante las librerías GAlib se ha hecho la implementación de la función objetivo. Todo este trabajo se ha desarrollado con software libre bajo el sistema operativo Linux.

\section{REFERENCIAS}

Belmans, R., R. D. Findlay y W. Geysen, A Circuit Approach to Finite Element Analysis of a Double Squirrel Cage Induction Motor, IEEE (1990).

Boglietti, A., A. Cavagnino y M. Lazzari, Experimental High-Frecuency Parameter Identification of AC Electrical Motors, IEEE Trans. On Industry Applications, 42(1), Enero/Febrero (2007).

Çunkas, M. y R. Akkaya, Design optimization of induction motor by genetic algorithm and comparison with existing motor, Mathematical and Computational Applications, 11(3), 193-203 (2006).

Çunkas, M., R. Akkaya y O. Bilgin, Cost optimization of submersible motors using a genetic algorithm and a finite element method, Int. J. Adv. Manuf. Technol., Springer-Verlag, Londres (2006).

Meeker, D., Induction Motor Example. IEEE (2002).

Mirafzal, B., G. L. Skibinsky y R. M. Tallam, Determination of Parameters in the Universal Induction Motor Model, IEEE Trans. On Industry Applications, 45(1), Enero/Febrero (2009).

Molinar, D., y otros cuatro autores. Calculation of two-axis induction motor model parameters using finite elements, IEEE (1996).

Nerg, J., J. Pyrhonen y J. Partanen, Finite element modelling of the magnetizing inductance of an induction motor as a function of torque, IEEE M. Transactions, 40(4), 2047-2049, Julio (2004).

Phumiphak, T. y C. Chat-Uthai, Estimation of Induction Motor Parameters Based on Field Test Coupled with Genetic Algorithm, IEEE (2002).

Rasmus, K. U., Models for Evolutionary Algoritms and Their Applications in System Identification an Control Optimization, University of Aarhus (2003).

Salon, S.J., Finite Element Analysis of Electrical Machines, Kluwer Academic Publishers, Boston (1995).

Von Lücken Martínez, C. D., Algoritmos Evolutivos para Optimización Multiobjetivo: un Estudio Comparativo en un Ambiente Paralelo Asíncrono, Universidad Nacional de Asunción (2003).

Wall, M., GAlib documentation, http://lancet.mit.edu/galib-2.4/, MIT (2000).

Yamazaki, K., An Efficient Procedure to Calculate Equivalent Circuit Parameters of Induction Motor Using 3-D Nonlinear Time-Stepping Finite-Element Method, IEEE M. Transactions, 38(1), 1281-1284, Marzo (2002). 